Instituto Internacional de Investigación y Desarrollo Tecnológico Educativo INDTEC, C.A.

DOI: https://doi.org/10.29394/scientific.issn.2542-2987.2017.0.0.19.355-375

OAI-PMH: http://www.indteca.com/ojs/index.php/Revista Scientific/oai

\title{
Huerto Escolar como Estrategia Pedagógica de la Sustentabilidad en la Educación Ambiental (Proyecto en ejecución)
}

Autora: Nayr Del Valle Rivas Silva Universidad Pedagógica Experimental Libertador, UPEL nayr1810@hotmail.com Barinas, Venezuela

\section{Resumen}

Esta investigación se trazó como propósito a establecer el huerto escolar como estrategia pedagógica de la sustentabilidad en la educación ambiental con los estudiantes de la Escuela Básica Nacional "Sebastián Araujo Briceño" del Municipio Pedraza Estado Barinas; la naturaleza de la investigación es cualitativa, el método es la Investigación Acción. Para fines del presente estudio, como informantes participarán tres (03) docentes y tres (03) estudiantes que pertenecen a la institución y gozan de reconocida responsabilidad y compromiso en la Escuela Básica Nacional "Sebastián Araujo Briceño", la técnica empleada es la entrevista semiestructurada, y el instrumento la guía de entrevista. El análisis de la información se realizará mediante la codificación, categorización, triangulación y estructuración de teorías. Luego de implementar las actividades con el huerto escolar se aspira concluir que los niños y niñas contribuyan al cuidado del ambiente y mantener en armonía el equilibrio ecológico que debe reinar en cualquier espacio donde viven y conviven los seres humanos. De esta manera la institución educativa va a presentar un aspecto ambiental acorde con los principios ecológicos de educación ambiental inmersos en el Currículo Básico Nacional.

Palabras clave: huerto escolar; estrategia pedagógica; sustentabilidad; educación ambiental. 


\title{
School Board as a Pedagogical Strategy for Sustainability in Environmental Education \\ (Project execution)
}

\begin{abstract}
This research was designed as a purpose to establish the school garden as a pedagogical strategy of sustainability in environmental education with the students of the National Basic School "Sebastián Araujo Briceño" of the Pedraza Municipality Barinas State; The nature of research is qualitative, the method is Research Action. For the purposes of the present study, three (03) teachers and three (03) students who belong to the institution will participate as informers and enjoy recognized responsibility and commitment at the "Sebastián Araujo Briceño" National Basic School. The technique used is the semistructured interview, And the instrument the interview guide. The analysis of the information will be done through the codification, categorization, triangulation and structuring of theories. After implementing the activities with the school garden, it is hoped to conclude that children contribute to the care of the environment and maintain in harmony the ecological balance that should reign in any space where human beings live and coexist. In this way the educational institution will present an environmental aspect in accordance with the ecological principles of environmental education immersed in the National Basic Curriculum.
\end{abstract}

Keywords: school garden; pedagogical strategy; sustainability; environmental education.

Date Received: 03-11-2016

Date Acceptance: 25-01-2017 


\section{Introducción}

En las últimas décadas, el crecimiento poblacional de la humanidad ha sido vertiginoso, tanto así, que el equilibrio ecológico que los pueblos del mundo habían mantenido durante muchos siglos, se ha visto afectado de una manera alarmante, pues en una fracción muy corta de tiempo, comparado con el albergue que el planeta ha tenido de la humanidad durante tantos años, el hombre le ha causado al planeta, acciones negativas e irreparables que jamás se pensó que pudiera producirle.

Sin embargo, en la misma media en que han venido apareciendo este tipo de problemas en el ambiente de las sociedades del mundo, también han ido naciendo alternativas que han permitido contrarrestar estas situaciones y empezar de manera certera a formar al hombre en torno al comportamiento que ha de mantener ante el ambiente y todos los elementos vitales que él alberga y que en definitiva le permiten mantener la vida en el planeta, incluyendo la de los seres humanos.

Por ello, la educación ambiental se ha convertido en materia esencial desde los diferentes ámbitos de formación presentes en las sociedades del mundo. De ahí que, González (2012), afirma que la educación ambiental implica: "la consideración de una visión para revisar y cambiar las actitudes y aptitudes humanas con relación al ambiente, educando de esta manera para una nueva forma operativa entre la realidad que se vive" (pág. 93), Construyendo de esta manera, los verdaderos valores que permitirán tener la naturaleza que queremos a través de una correcta conciencia conservacionista.

\section{El Problema}

La educación ambiental por sí sola no resultaría eficiente, si no va acompañada de estrategias de sensibilización que les permita a los niños y niñas interactuar de manera directa con el ambiente, es así, como la educación 
ambiental constituye según Antúnez (2011), "uno de los elementos más revolucionarios del proceso educativo" (pág. 61), pues está dirigida a diagnosticar, proteger y conservar la naturaleza en los estudiantes de hoy día, que indudablemente serán los hombres del mañana que actuarán de acuerdo a su conciencia sobre el ambiente que los rodea para lograr el desarrollo sostenible y sustentable como fin supremo, y en esencia, para lograr un espacio sano y perdurable en el tiempo, y así heredar un mundo sano y apto para la vida de las personas.

Es así como, los huertos escolares se han venido convirtiendo en una estrategia ecológica de gran alcance en las instituciones educativas, pues tal como lo plantea Ordaz (2012), "los huertos escolares puedan constituirse en un modelo operativo viable, que posibilita la ejecución de acciones dirigidas específicamente a la consecución efectiva de las metas propuestas a nivel institucional y organizacional" (pág. 1). Es importante destacar que durante años se ha visualizado a nivel educativo la necesidad de crear huertos escolares como alternativa para estimular a los estudiantes a la actividad agrícola, sabiendo la importancia que tienen esta estrategia de tipo ambientalista para formar a los estudiantes, no desde lo teórico, sino desde la practica misma del contacto directo con el ambiente.

Cabe destacar, que en Venezuela ha venido adelantando certeros esfuerzos en materia de educación ambiental ya que por parte del Ministerio del Poder Popular para la Educación (2007), en materia de educación ambiental, se ha insertado en el Currículo Nacional Bolivariano el eje integrador ambiente y salud integral, elevándose a una situación específica, por medio de los proyectos de aprendizaje, ya que no se presentan actividades concretas que vayan en función de fortalecer la actitud conservacionista en docentes y estudiantes para profundizar la conciencia ecológica de los mismos. 
De esta manera, Marcano (2010), plantea que el la educación venezolana "se ha promovido en el proceso diario de la enseñanza de educación ambiental el respeto al entorno natural donde se desenvuelven docentes, niños y niñas y su interacción equilibrada con la naturaleza" (pág. 92); por cuanto la realidad plasmada en programas, planes y proyectos son evidentes los problemas que enfrentan las comunidades con respecto al deterioro de su ambiente escolar y comunitario producto de conductas anti ambientalistas y por ende antiecológicas, más la ausencia de actitudes favorecedoras para el éxito de la enseñanza y el aprendizaje de las ciencias ambientales. Por ello, Pereira (2012) argumenta que:

Educar en el trabajo supone asumir en serio el principio pedagógico del aprender haciendo, de convertir el aula en el taller donde se construyen conocimientos a partir de las experiencias, intereses y saberes de los estudiantes. Asimismo, es necesario enseñar a aprovechar bien el tiempo, resolver cooperativamente y creativamente los problemas, seguir instituciones y procesos, buscar calidad en los productos, valorar el trabajo responsable y reconocer que debe estar al servicio de los seres humanos (pág. 4).

Partiendo de estos planteamientos es importante resaltar que la investigadora a través de la observación directa y conversaciones con algunos docentes y representantes de la Escuela Básica Nacional "Sebastián Araujo Briceño", ha podido evidenciar conductas antiecológicas e los niños y niñas, que llevan al deterioro del ambiente causando desarmonía en el equilibrio ecológico que debe reinar en cualquier espacio donde viven y conviven los seres humanos.

Estas causas están centradas en daños a los elementos naturales que se tienen en la institución, como lo es el deterioro del jardín de la escuela, la proliferación de basura en el espacio escolar, el daño a los animales que de manera natural tienen su nicho ecológico el algunas plantes o espacio verde 
presente en la escuela. Estas causas han traído como consecuencias, que la institución no presente un aspecto ambiental acorde con los principios ecológicos de educación ambiental inmersos en el Currículo Básico Nacional, que los niños no tengan la sensibilidad acorde para respetar los elementos naturales presentes en la escuela.

Estas causas y consecuencias, han motivado a la investigadora para el desarrollo de la presente investigación que busca establecer el huerto escolar como estrategia pedagógica para la sustentabilidad de la educación ambiental con los estudiantes de la Escuela Básica Nacional "Sebastián Araujo Briceño" del Municipio Pedraza Estado Barinas. Surgiendo así las siguientes interrogantes:

¿Qué conocimientos tienen los niños y niñas de la Escuela Básica Nacional "Sebastián Araujo Briceño" en torno al desarrollo de los huertos escolares?

¿Cuál es la aceptación que tienen los niños y niñas de la Escuela Básica Nacional "Sebastián Araujo Briceño" en torno a la implementación del huerto escolar como estrategia pedagógica para la sustentabilidad de la educación ambiental?

¿Qué elementos será preciso tener en cuenta para el establecimiento del huerto escolar como estrategia pedagógica en la sustentabilidad de la educación ambiental con los estudiantes de la Escuela Básica Nacional "Sebastián Araujo Briceño"?

\section{1. Propósitos de la Investigación}

\section{2. .1.1. Propósito General}

Establecer el huerto escolar como estrategia pedagógica para la sustentabilidad de la educación ambiental con los estudiantes de la Escuela 
Básica Nacional "Sebastián Araujo Briceño" del Municipio Pedraza Estado Barinas.

\section{Fundamentación Teórica}

La fundamentación teórica es representada por el procedimiento mediante el cual se recopila la información que se requiere para sustentar conceptualmente una investigación. Por lo tanto, Farci y Ruiz (2014) en su guía para su planificación, ejecución y comunicación exponen:

Una fundamentación bibliográfica no es un mero reporte de la bibliografía revisada. Es un texto procesado, redactado de tal manera que en él, el investigador sustenta su trabajo, define sus eventos y expone la teoría de la cual va a partir, se apoya en autores para soportar o darle fuerza a sus planteamientos. El eje de la fundamentación es el esquema conceptual del investigador. (pág. 35)

Por lo citado, esta fase tiene el propósito de dar al estudio un sistema coordinado y coherente de proposiciones que permitan abordar de forma clara el problema. De éste dependerá el resultado del trabajo, permitirá en la investigadora crear sus propias posturas, asumir las derivaciones de investigaciones anteriores y esforzarse por orientar el trabajo de un modo coherente, situar el problema que se está estudiando dentro de un conjunto de conocimientos, permitiendo orientar la búsqueda y ofrecer una conceptualización adecuada de los términos que se utilizaran en la investigación.

\subsection{Huerto Escolar}

El huerto escolar, es un área de cultivo de hortalizas, granos, cereales y frutas entre otros, dentro de un espacio escolar o lugar próximo a la escuela. De acuerdo con lo señalado por el Ministerio del Poder Popular para la Educación (2015: 54); se constituyen en gran utilidad desde la experiencia 
educativa, esta idea está plasmada en las orientaciones educativas para los niveles y modalidades del subsistema de educación Básica entre sus planes, programas y proyectos, específicamente en el proyecto Todas las manos a la siembra de educación básica, donde aborda los contenidos referentes a la cultura agroecológica en el proceso curricular del eje integrado Ambiente y salud integral.

Se trata de una experiencia educativa que busca transferir a los diversos actores de la comunidad el conocimiento de tecnologías orgánicas y de reciclaje para la producción de hortalizas y frutas para el uso comunitario. Asimismo, se utiliza el huerto como taller o laboratorio en las áreas del conocimiento. De acuerdo a estos señalamientos, los educandos tienen la misión de aprender con cursos especiales sobre los cultivos y los docentes de no desaprovechar esta forma de enseñar y aprender.

\subsection{Manejo del Huerto}

En esta fase es conveniente, contar con suficiente agua, abonos, población y terreno. En relación al abono, el principal abono lo componen los estiércoles de los animales, pero de no tenerlo se puede hacer uso de abonos orgánicos, tales como; harina de pescado, de carne o de algas y otro elemento nitrogenado. Se debe evitar en lo posible usar nitrógeno artificial o inorgánico porque elimina los elementos naturales que contiene el suelo. El abono orgánico se puede adquirir recolectando los desechos vegetales y alimenticios.

Se observa que, al abordar el establecimiento del huerto escolar como estrategia pedagógica para la sustentabilidad de la educación ambiental con los estudiantes de la Escuela Básica Nacional "Sebastián Araujo Briceño" del Municipio Pedraza Estado Barinas; se tiene que en el mismo pueden cultivarse plantas medicinales que contribuyan a mejorar y mantener el cuerpo en un estado saludable, como lo es el jengibre, romero, manzanilla entre otros. Así mismo pueden considerarse, las plantas que se utilizan como condimentos 
cebollas, perejil, cilantro y otras; plantas ornamentales como margaritas claveles, entre otras, también plantas comestibles que sean de fácil cuidado como auyamas, acelgas y lechugas. Con el fin de contribuir en el desarrollo Social y Personal de los niños y niñas.

Sobre este particular, el huerto en la escuela debe constituirse en un potencial de aprendizaje, en tanto que favorezca el estudio desde el área de ciencias naturales de los factores ambientales, así como la relación de la cadena alimentaria que se establece entre los seres vivos; es importante destacar que en los ambientes propios de la escuela los estudiantes puedan aprender a diferenciar los factores bióticos y abióticos presentes en él. Así mismo, un huerto escolar combinado con lecciones de nutrición no sólo instruye a los estudiantes, sino también a sus familias sobre la importancia de una alimentación saludable y variada.

Por consiguiente, resulta bastante claro inferir que el huerto escolar es una preparación para la vida adulta; en la medida en que se ejerce para lograr algo, hay que proponer, exigir, ceder, pautar reglas claras y establecer estrategias. El huerto es una fuente de aprendizaje para la comunidad de padres y representante, niñas y niños. Al hacer referencia al huerto escolar y el currículo se tiene que el mismo es el marco idóneo para trabajar los ejes transversales, especialmente el eje educación ambiental, pues en él tienen cabida temas como el consumo, la alimentación, la basura, el reciclaje, la salud, el desarrollo endógeno y valores como la apreciación de todas las formas de vida, la equidad y la solidaridad para con las demás personas y el planeta.

Así, por ejemplo, en el área de ciencias de la naturaleza, donde se propone el enfoque sistémico de la naturaleza, en el que se enfatizan las interacciones que determinan la organización o estructura del sistema y los cambios o estados de equilibrio, el acondicionamiento y el estudio del huerto escolar resulta un recurso didáctico de primer orden para estudiar la diversidad 
de la naturaleza, los componentes de un ecosistema, las transferencias energéticas, la acción transformadora de los seres humanos en la naturaleza y nuestra responsabilidad en los desequilibrios que ocurren en la naturaleza. Por tanto, se pueden estudiar en esta área, el ciclo vital de las plantas, ciclo del agua, clima, tiempo atmosférico, tipos de terreno, tipos de paisaje, fauna común de un huerto y otros aspectos que se consideren convenientes 0 apropiados.

A través de las actividades del huerto se pueden estudiar las relaciones tecnología-sociedad en sus dos sentidos, la tecnología como factor de progreso o de transformación cultural (introducción de los tractores, abonos.), o la tecnología como herramienta controlada por las élites y por lo tanto fuera del alcance de las decisiones autónomas de la ciudadanía (comercio internacional, alimentos transgénicos, entre otros.).

En el área de ciencias sociales, geografía e historia se puede abordar el estudio del huerto desde una perspectiva geográfica, económica y sociológica, estudiando las transformaciones y utilizaciones de la naturaleza, los sistemas de explotación agraria y las problemáticas asociadas: excedentes, hambre, degradación de los suelos; y las alternativas al desarrollo desigual: eco-desarrollo, agricultura biológica, desarrollo sostenible en el mundo rural, entre otros.

En matemáticas, se pueden trabajar mediciones diversas (altura de las plantas, marco de plantación, diámetros). Porcentajes: conteo de semillas sembradas y germinadas, número de plantones, peso, problemas, entre otros.

En el área de lenguaje, se puede trabajar conocimiento de vocabulario nuevo, adivinanzas, cuentos populares, lectura de textos y redacciones. Entre tanto en el área de artística o artes plásticas, se puede desarrollar por medio de murales, dibujos, mosaicos con semillas, hojas, palitos o piedrecitas; modelado en plastilina o material similar de frutas, verduras y pequeños animales. De igual forma, canciones populares, diferentes ritmos con piedras 
y palos a modo de claves, elaboración de instrumentos como maracas. En el área de educación física fácilmente se pueden desarrollar: circuitos, transporte de mangueras, cubos de agua en cadena, labrado de la tierra, aporcado, entre otras actividades a considerar.

\subsection{Educación Ambiental}

Según Hernández (2012), la Educación Ambiental la entendemos como un:

Proceso continuo, interactivo e integrador, mediante el cual el ser humano adquiere conocimientos y experiencias, los comprende y analiza, los internaliza y los traduce en comportamientos, valores y actitudes que lo preparen para participar protagónicamente en la gestión del ambiente y el desarrollo sustentable. (pág. 73)

En este sentido, se considera que el desarrollo sostenible tiene el compromiso y la oportunidad de colocar respetuosamente a los seres vivos (incluidos los seres humanos y sus culturas) en el centro de la actividad productiva y entender el proceso económico como parte de un sistema abierto que mantiene relaciones permanentemente con otros sistemas, entre ellos el ambiental. El propósito de redención de la pobreza y superación de la exclusión, mediante la puesta en práctica de formas de producción creativas, fórmulas de organización social solidario, tecnologías sociales y ecológicamente apropiadas y el estímulo a estilos de vida y consumo más equilibrados y justos.

Asimismo, Piñango (2013), establece que "A través de la Educación Ambiental se pretende que individuos y colectivos manejen una serie de conocimientos e información para que puedan desarrollar una actitud crítica y positiva hacia nuestro entorno, para que podamos mejorar la calidad de vida colectiva" (pág. 83). Sabiendo que la calidad de vida es el grado de bienestar individual y en grupo, derivado de la satisfacción de las necesidades básicas de la población en educación, salud, vivienda y servicios básicos, recreación, 
cultura y deportes, trabajo, participación, accesibilidad y ambiente físico, para asegurar su existencia, permanencia y trascendencia en un espacio dado y en un momento determinado.

\section{Referente Metodológico}

\subsection{Enfoque de la Investigación}

De acuerdo a los objetivos planteados el presente estudio se enmarca en el enfoque cualitativo porque tal como lo expresa Martínez (2014), la investigación trata de aproximarse a la realidad concreta del problema con miras a su transformación y las aplicaciones para la solución, por lo que "destaca el registro objetivo de los hechos en el momento de sucederse, generando acciones determinantes con el grupo social en estudio" (pág. 84).

Por otra parte, Murcia, (citado en Hurtado y Toro 2011), afirma que en el enfoque cualitativo "el investigador se inserta dentro de la realidad social de los grupos y comunidades para contribuir con la interpretación objetiva de la misma y buscar junto con ellos, la formulación de acciones para transformarlas" (pág. 53). Indicando con ello, que el presente estudio está dentro de esta línea del enfoque cualitativo.

\subsection{Tipo de Estudio}

Es importante destacar que en el presente estudio el tipo de investigación está enfocada en el tipo de investigación acción, la cual se desarrollará en la Escuela Básica Nacional "Sebastián Araujo Briceño" del Municipio Pedraza Estado Barinas, buscando Establecer los huertos escolares como estrategia pedagógica para la sustentabilidad de la educación ambiental en los estudiantes de la Escuela Básica Nacional "Sebastián Araujo Briceño" del Municipio Pedraza Estado Barinas. Se utilizará como apoyo la fenomenología, la cual parte de elementos fundamentales para dar garantía al cumplimiento de los objetivos planteados por la investigadora y dar solución al problema 
utilizando los métodos necesarios para el estudio y análisis de la realidad que aquejan al sector escolar. Al respecto Martínez (2014) expresa que:

La investigación- acción realiza simultáneamente la expansión del conocimiento científico y la solución de un problema, mientras aumenta, igualmente, la competencia de sus respectivos participantes (sujetos coinvestigadores) al ser llevada a cabo en colaboración, en una situación concreta y usando la realimentación de la información en un proceso clínico. (pág. 239).

La aplicación de la investigación acción permitirá dar solución a la problemática presente en la institución, esto generará una serie de pasos para llevar a cabo los objetivos planteados, a tal fin se ejecutarán las diversas acciones para lograr los objetivos planteados.

\subsection{Diseño de la investigación}

Siendo el estudio de tipo investigación acción participativa, de acuerdo al nivel de profundidad del estudio, está enmarcado en un diseño de campo, que, según el Manual de Trabajos de Grado de Especialización y Maestría y Tesis Doctorales, de la Universidad Pedagógica Experimental Libertador UPEL (2014) la define como:

Es el análisis sistemático de problemas en la realidad con el propósito bien sea de descubrirlos, interpretarlos, entender su naturaleza y factores constituyentes, explicar sus causas y efectos, o predecir su ocurrencia, haciendo uso de métodos característicos, de cualquiera de los enfoques de investigación conocidos o en desarrollo. Los datos de interés son recogidos en forma directa de la realidad... (pág. 18).

Por cuanto permitirá la recolección de datos directamente de la realidad donde ocurren los hechos, en donde se presenta una problemática de una institución educativa, teniendo como establecer los huertos escolares como 
estrategia pedagógica para la sustentabilidad de la educación ambiental en los estudiantes de la Escuela Básica Nacional "Sebastián Araujo Briceño" del Municipio Pedraza Estado Barinas, partiendo del diagnóstico que permita verificar si los actores del proceso presentan debilidades, oportunidades, amenazas y fortalezas en el desarrollo del mismo. Para darle a la investigadora la información necesaria que permitirá profundizar en las unidades de análisis.

Cabe resaltar que el diseño de la investigación se llevará a cabo en las siguientes fases:

4.3.1. Fase 1. Diagnóstico. Consiste en identificar los informantes, en el grupo de docentes y estudiantes de la Escuela Básica Nacional "Sebastián Araujo Briceño" del Municipio Pedraza Estado Barinas, con la finalidad de señalar las diferentes cualidades necesarias para la investigación, por otra parte, se usará la observación directa en el grupo de estudio el cual establece el enfoque cualitativo.

Según Martínez (2014). “...Para ello, el investigador vive lo más que pueda con las personas, y solo lo será en la medida en que sea percibido como una "buena persona", franca, honesta, inofensiva y digna de confianza" (pág. 89). En este sentido, las observaciones realizadas permitirán a la investigadora establecer contacto con los informantes, interactuando con el entorno y usando al mismo tiempo notas de campo.

Por otra parte, se aplicará la entrevista en profundidad a los informantes donde se les preguntará a los docentes y estudiantes sobre diferentes tópicos que permitan determinar el nivel de conocimiento que tienen en relación a los huertos escolares como estrategia pedagógica para la sustentabilidad de la educación ambiental, accediendo a expresar de forma libre las opiniones referentes al tema de investigación. Para la planificación de la entrevista se realizarán las preguntas y se clasificarán los temas con anterioridad con el fin de evitar la improvisación y vacíos en la conversación. 
Luego de tener la información se procederá a realizar la categorización de los informantes, la cual consistirá en un primer momento en la lectura detallada, el análisis, y la decodificación. Después de haber desarrollado lo antes mencionado se dará el proceso de triangulación de la información tomando como base las categorías por cada informante, teorías resaltantes de la investigación y la interpretación de la investigadora, con la finalidad de poder generar la teorización que es la argumentación final, siendo coherente y lógico integrando todos los resultados obtenidos de la información.

4.3.2. Fase 2. Plan acción. En esta fase se diseñará el plan acción, como resultado de la información recogida en el diagnóstico, por consiguiente, el diseño se reflejará por un conjunto de actividades que servirán para desarrollar las actividades relacionadas con establecer los huertos escolares como estrategia pedagógica para la sustentabilidad de la educación ambiental en los estudiantes de la Escuela Básica Nacional "Sebastián Araujo Briceño". Las actividades diseñadas se realizarán pensando en las necesidades que presentan los docentes, tomando en cuenta la disposición, recursos y tiempos disponibles que le permitieran a los involucrados participar de forma activa en las actividades de incorporación.

4.3.3. Fase 3. Ejecución del plan acción. Consistirá en llevar a cabo la ejecución de las actividades lúdicas bajo una programación estableciendo referente a estrategias, contenido, actividades, día, fecha, recursos, responsables y acciones en los y las docentes y estudiantes de la Escuela Básica Nacional "Sebastián Araujo Briceño". La aplicación se desarrollará en los entornos de la escuela, propuesta por los involucrados y la investigadora quien será la responsable de llevar a cabo los objetivos propuesto en la investigación acción.

4.3.4. Fase 4. Valoración del plan acción. Permitirá valorar las actividades aplicadas, programadas en el plan acción, donde se describirá el impacto que tuvo las acciones implementadas durante la investigación, bajo el 
enfoque cualitativo, donde la investigadora expresará las vivencias observadas en la puesta en práctica de cada actividad lúdica aplicada al grupo. Asimismo, se explicará detalle a detalle la experiencia tanto positiva como negativa de las actividades para establecer los huertos escolares como estrategia pedagógica para la sustentabilidad de la educación ambiental en los estudiantes de la Escuela Básica Nacional "Sebastián Araujo Briceño".

\subsection{Informantes Calves}

Dado que la investigación cualitativa busca la interpretación y comprensión de los fenómenos, para lo cual se pone de manifiesto la descripción y análisis de acciones y vivencias de los individuos, Martínez (2012), recomienda que se "requiere trabajar con muestras pequeñas para facilitar una investigación lo más cercana posible a la realidad" (pág. 135). Por eso se habla de participantes o informantes, quien ha de ser una persona capaz de aportar información sobre el elemento de estudio.

Es de acotar que Vera y Ponce (2012), en el proceso investigativo, la identificación de los participantes, generalmente "es una muestra seleccionada, no aleatoria; ya que el investigador procura por una que concierne más a los propósitos de la investigación" (pág. 23). Considerando lo expresado por los mencionados autores, para fines del presente estudio, como informantes participarán tres (03) docentes y tres (03) estudiantes que pertenecen a la institución y gozan de reconocida responsabilidad y compromiso en la Escuela Básica Nacional "Sebastián Araujo Briceño". Cada uno ha de ofrecer información relevante en cuanto a las unidades de análisis planteadas en el presente estudio.

\subsection{Técnicas e Instrumentos de Recolección de datos}

Las técnicas para la recolección de datos, son los distintos procedimientos, formas o maneras que se emplean al recoger información. 
Según Vallejo (2011) expresa que "existe variedad de técnicas e instrumentos de recogida de datos de acuerdo a la temática a investigar, los objetivos propuestos, la adecuación a cada situación y su mayor o menor conocimiento" (pág. 150). Es así como, las técnicas de investigación representan un elemento clave para el investigador en el proceso de recolección de la información.

A tales fines, Pérez (2011), manifiesta que, entre las técnicas de recolección de datos en una investigación cualitativa, figuran las "de papel y lápiz, notas de campo, el diario, anécdotas, comentarios, entrevistas y grabaciones entre otras" (pág. 156). Es de agregar que la mencionada autora, expresa que las técnicas de recolección de datos en la investigación cualitativa son diversas; porque se trata de obtener el máximo de información pertinente al estudio, que genere interpretación más aproximada de la realidad y de los factores incidentes en ella. Por tanto, en este caso se utilizará como técnica la entrevista y la observación directa.

En cuanto a las entrevistas las mismas constituyen una estrategia clave para la recopilación de los datos para el estudio que se realiza. De acuerdo a Báez (2011), en la entrevista "el investigador prepara de antemano una lista de preguntas; pero sin limitar la misma a ciertas preguntas, ni fijar previamente el orden en las que se contestan" (pág. 21). Por tanto, su uso permitirá sondear las respuestas y desarrollar temas que surgen en el transcurso de la entrevista, aclarar términos, definir problemas, orientar, motivar al entrevistado, elevar su nivel de interés y colaboración y estimular su memoria.

Asimismo, Ramírez (2012) expresa que la observación participante "es cuando para obtener los datos el investigador se incluye en el grupo, hecho o fenómeno observado, para conseguir la información desde adentro" (pág. 112). Para la presente investigación, la investigadora actuará desde dentro, pues forma parte del grupo de docentes y estudiantes que representan el campo de investigación. 
En lo que respecta a los instrumentos, Báez (2011), señala que éstos "consisten en formularios diseñados previamente para registrar la información durante el proceso de recolección de datos" (pág. 154). Es de referir que para esta investigación se empleará como instrumento una guía de entrevista semi estructurada para abordar a los docentes de aula y una guía de observación para registrar las observaciones hechas.

\subsection{Validez y Fiabilidad de los Instrumentos}

Cabe agregar que la guía de entrevista y guía de observación como instrumentos no serán sometidos a procesos de validación mediante expertos, por cuanto no se trata de cuantificar los hechos, sino más bien comprender mejor la naturaleza del fenómeno, acerca de establecer los huertos escolares como estrategia pedagógica para la sustentabilidad de la educación ambiental en los estudiantes de la Escuela Básica Nacional "Sebastián Araujo Briceño" del Municipio Pedraza Estado Barinas.

En este orden de ideas, puede decirse que para la validación del instrumento que se aplicará será interna, puesto que tal como lo refiere Pérez (2012), se garantizan por la aplicación de procesos holísticos de la investigación, entrevistas; triangulación de las diversas fuentes de datos, permitiendo que todos los involucrados se expresen y aporten variedad de información que no se puede obtener mediante preguntas solamente cerradas.

Al efecto, la mencionada autora agrega, que la validez de los instrumentos "es el grado en que las respuestas son independientes de las circunstancias accidentales de la investigación y en la medida de que sea válido lo que se interpreta en forma correcta" (pág. 157). En consecuencia, la validez, se sustenta en la forma cómo se recogerán los datos necesarios para la mejor comprensión del fenómeno estudiado, la captación de los momentos, se analizan e interpretan los acontecimientos dentro de su propio contexto. 
Además, es de considerar que tal como lo señala Martínez (2012), en la investigación cualitativa se habla de "fiabilidad de la investigación misma" (pág. 86). Además, en opinión del autor, los instrumentos utilizados para recabar información se constituyen en elementos que fortalecen, testimonian y respaldan el estudio realizado. Puesto que la fiabilidad constituye el grado en que las respuestas son independientes de las circunstancias accidentales de la investigación.

De allí, que para garantizar la fiabilidad de la investigación, el mencionado autor, sugiere utilizar categorías descriptivas concretas y precisas, con datos primarios y no manipulados; igualmente, solicitar la colaboración de los informantes para constatar la objetividad de las notas de campo y, hacer uso de instrumentos de investigación que evidencien la realidad estudiada a través de imágenes y sonidos para que pueda ser verificada y analizada posteriormente por otros investigadores o evaluadores de la investigación.

\section{Conclusiones}

Es ineludible concluir que existe la perentoria necesidad de repensar nuestra manera de pensar para poder llegar al fondo del problema, pues de no hacerlo todos los esfuerzos que se hagan por salvar y por ende conservar el medio ambiente serian pañitos de agua tibia tiene como objetivo fundamental crear una nueva conciencia, en primer lugar, que conlleve a la unidad familiar.

Los huertos escolares son una alternativa ecológica educativa por excelencia, debido a que en el niño aprende a cultivar de manera sana los alimentos que requiere para su alimentación, investiga y comprende la importancia de transformar el modelo agrícola actual.

De igual manera existe la necesidad de enseñar al niño una educación ambiental apoyada en estrategias significativas donde ponga todas sus 
capacidades para entender y comprender mejor la necesidad de cambio en la cultura productiva y ambientalista que existe en el mundo actual. Se deben realizar estrategias como la proyección de videos en el cual se puedan visualizar la experiencia que han tenido otras personas. Así como también videos que proyecten la perentoria necesidad de cuidad el medio ambiente.

\section{Referencias}

Antúnez, N. (2011). La Formación de Brigadas Ambientales para la Protección del Ambiente en la Escuela rural Concentrada La Llovizna. Adscrita al NER 612 del Municipio Antonio José de Sucre. Trabajo de grado. Barinas, Venezuela: UNELLEZ, Postgrado.

Báez (2011). El proyecto de Investigación. Caracas: Episteme.

González, L. (2012) La Preocupación por la calidad del Medio Ambiente.

Un modo cognitivo sobre la conducta ecológica. Madrid, España:

Universidad Complutense de Madrid. Tesis de Doctorado.

Hurtado I., y Toro J. (2007). Paradigmas y Métodos de la Investigación en

tiempos de cambios. Venezuela: Editorial CES, S.A.

Martínez (2012). Nuevos paradigmas en la investigación. (1ra. ed.). México: MacGraw- Hill.

Martínez (2014). La Investigación Cualitativa Etnográfica en Educación. México: Editorial Tillas.

Ordaz D. (2012). El huerto escolar como herramienta didáctica para el desarrollo productivo en la escuela. Trabajo de grado. Recuperado de: http://saber.ucv.ve/ispui/bitstream/123456789/6287/1/Tesis.pdf UPEL (2014). Manual de trabajo de grado de Especialización y Maestrías y tesis doctorales. (4ta. Edición). FEDUPEL. Caracas, Venezuela. 


\title{
Nayr Del Valle Rivas Silva
}

\author{
e-mail: nayr1810@hotmail.com
}

Técnico Superior en Educación Integral (Instituto

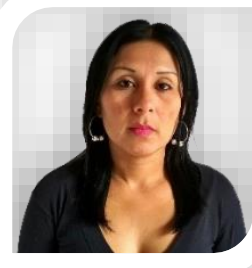

Universitario de tecnología Coronel Agustín Codazzi, Barinas). Licenciada en Educación Integral (Universidad Católica Cecilio Acosta, Maracaibo). Magíster en Educación Ambiente y Desarrollo Universidad Pedagógica Experimental Libertador (FALTA). Docente titular (Escuela Básica Nacional Sebastián Araujo Briceño).

\footnotetext{
El contenido de este manuscrito se difunde bajo una Licencia de Creative Commons ReconocimientoNoComercial-Compartirlgual 4.0 Internacional
} 